\title{
Aeromonas hydrophila Panophthalmitis in Pemphigus Vulgaris: A Case Report
}

\author{
Sukhumal Thanapaisal Patsuda Thariya \\ KKU Eye Center, Department of Ophthalmology, Khon Kaen University, \\ Khon Kaen, Thailand
}

\section{Keywords}

Endophthalmitis · Panophthalmitis · Aeromonas hydrophila $\cdot$ Pemphigus vulgaris

\begin{abstract}
A 47-year-old Thai man, who had recently been diagnosed with active pemphigus vulgaris (PV) and treated with oral prednisolone together with intravenous dexamethasone, presented with severe ocular pain and light perception vision of the left eye for 4 days. Ophthalmic examination revealed periorbital soft tissue swelling with marked intraocular inflammation in the left eye. Severe panophthalmitis was diagnosed. Enucleation was performed after failure of intravenous antibiotics administration. Vitreous culture revealed Aeromonas hydrophila but no primary source of infection was clearly identified. An immunocompromised status accompanied with corticosteroid treatment is a risk factor for septicemia. A. hydrophila panophthalmitis was detected in the PV patient, and this organism caused a rapid disease progression with poor visual prognosis.

(c) 2019 The Author(s)

Published by S. Karger AG, Basel
\end{abstract}

\section{Introduction}

Ocular involvement in pemphigus vulgaris (PV) is uncommon. Autoimmune antibodies in these patients cause blisters on the skin and mucosa, resulting in a noninfective condition. Although ocular infection in PV patients is rare, the immunocompromised status in these 
patients with other medical conditions results in increased susceptibility to infection. Fungal keratitis and bilateral herpetic keratitis associated with corticosteroid treatment have been reported as ocular infection in pemphigus patients [1-3].

Herein, we report the case of a 47-year-old man with recently diagnosed PV who presented with severe panophthalmitis caused by Aeromonas hydrophila.

\section{Case Report}

A 47-year-old Thai man presented with severe ocular pain and decreased vision in the left eye lasting for 4 days. He had been diagnosed with active PV 3 weeks earlier, for which he had been treated with oral prednisolone for 2 weeks. Because of the only slight improvement of the skin lesions, the patient then was treated by intravenous dexamethasone $5 \mathrm{mg}$ every $6 \mathrm{~h}$ ) with prophylaxis doses of intravenous cloxacillin ( 1 g every $6 \mathrm{~h}$ ) for 1 week during hospitalization. Although there was a significant improvement of the skin lesions, the patient started to report ocular pain and blurred vision in the left eye. The pain, redness, and swelling around the upper and lower eyelids progressed so rapidly that the patient could not open his eye. The vision reduced to light perception in 4-day time. After stepping the antibiotics up to intravenous imipenem ( $500 \mathrm{mg}$ every $6 \mathrm{~h}$ ), his symptoms gradually worsened. Then, he was referred to our hospital.

Physical examination on admission revealed high body temperature $\left(38.1^{\circ} \mathrm{C}\right)$ as well as skin lesions with multiple discrete flaccid blisters and oval-shaped erosions covered with hemorrhagic crust distributed along the trunk and extremities. Some of the lesions had healed as postinflammatory hyperpigmented patches without evidence of cellulitis or pustules (Fig. 1). On ophthalmic examination, periorbital erythema and marked soft tissue swelling with hyperpigmented flaccid bullous were noted at the left upper eyelid (Fig. 2). His visual acuity was reduced to light perception with poor light projection in the left eye and 20/30 in the right eye. Intraocular pressure in the left and right eyes were 46 and $16 \mathrm{~mm} \mathrm{Hg}$, respectively. Slit-lamp examination demonstrated severe chemosis with a yellowish discharge, totally haze cornea, a generalized shallow anterior chamber with marked anterior chamber reaction, $2 \mathrm{~mm}$ of hypopyon, and plasmoid formation. The pupil of the left eye was slightly reactive to light and positive for relative afferent pupillary defect. The patient's fundoscopic view was obscured. The extraocular muscles were limited in all directions of gaze. The right eye was unremarkable.

Ultrasonography of the left eye showed generalized opacity of the vitreous and chorioretinal thickening with no evidence of retinal abscess (Fig. 3). A computed tomography scan demonstrated left proptosis with periorbital soft tissue swelling, diffused scleral thickening, and retrobulbar fat stranding compatible with orbital cellulitis (Fig. 4). The paranasal sinuses were normal. No subperiosteal abscess was documented.

Laboratory examination of renal and liver function was normal. The urinalysis showed no infection. Anti-HIV and diabetic test were negative. Blood culture and skin biopsy results were negative for organisms. Dental examination, nasal endoscopy, and liver ultrasonography were conducted but failed to identify the primary source of infection.

The patient was diagnosed infective panophthalmitis. He was treated with intravenous ceftazidime and vancomycin. Despite the stepping up of antibiotics, the condition worsened. Risks, benefits, and alternatives to pars plana vitrectomy and intravitreal antibiotics injection were extensively discussed. Due to the severe painful eye with extensive orbital lesion, poor visual prognosis after surgery, and low socioeconomic status, the patient finally made the 
decision to undergo enucleation with glass ball implantation. Moderated growth of $A$. hydrophila was isolated from the vitreous culture and was susceptible to ceftazidime, gentamicin, and ciprofloxacin. The systemic administration of antibiotics was continued for 10 days then stepped down to oral administration.

The infection was controlled after the enucleation. High doses of oral prednisolone, dapsone and azathioprine were administered in order to treat the active PV. Fortunately, no evidence of fellow eye infection was detected after 1 year of follow-up.

\section{Discussion}

$\mathrm{PV}$ is an autoimmune mucocutaneous blistering disorder affecting the skin and mucous membranes. The production of autoantibodies against desmosomal adhesion molecules (desmoglein) leads to flaccid blisters [4]. Ocular involvement in PV is uncommon. Therefore, many cases are not detected due to the presence of only minimal symptoms. Bilateral conjunctivitis remains the most common finding in these cases, followed by blepharitis [4-8]. The prognosis in noninfective cases is good, but in the infective cases usually result in poorer visual outcomes due to the immunocompromised status of these patients.

A. hydrophila is a facultative anaerobe Gram-negative rod found in water, soil, and drinking water [9]. This organism could be also detected in fresh fish, fresh vegetables, and dairy products [10]. In 1988, in a population-based study performed in California, USA, a total of 280 individuals experienced $A$. hydrophila infection [11]. The gastrointestinal tract (81\%) was found to be the most common source, followed by wound infections (9\%) [11]. In patients with acute diarrhea, $A$. hydrophila was reported in 6.5 and $6.9 \%$ in India and Hong Kong, respectively $[12,13]$. A high number of skin soft-tissue infection was reported during the Tsunami devastation in Thailand [14] and Hurricane Katrina in New Orleans, LA, USA [15]. A. hydrophila was also isolated from household drinking water in South Africa [16].

The organism has been found to be a highly pathogenic opportunistic infection in immunocompromised hosts, especially in liver cirrhosis and malignancy [17]. Gastroenteritis, peritonitis, cholangitis [18], liver abscess [19], cellulitis, and pneumonia [20, 21] could lead to bacteremia in patients with predisposing medical conditions. Nosocomial bacteremia could develop within the first week of hospitalization, and the isolates in some strains were less susceptible to cefoxitin and cefotaxime than community-acquired condition [17]. This organism has been reported for ocular infection including conjunctivitis [22, 23], traumatic corneal ulcer, contact lens-related corneal ulcer [23], and traumatic endophthalmitis [24]. Endogenous endophthalmitis [25-27] and orbital cellulitis [28] could be found in immunocompromised patients; malignancy, diabetes mellitus, prolonged central venous line for parenteral nutrition, and myelodysplastic syndrome, as well as those who experienced septicemia. In endophthalmitis, A. hydrophila showed clinical signs of severe endophthalmitis with rapid progression and destructive lesions compared to other common pathogens. No main specific sign was reported for this organism so far.

We hypothesized that our patient developed infective endophthalmitis, then progressed to panophthalmitis. Inflammation in patients with endophthalmitis alone usually confines in the intraocular tissue while secondary preseptal and septal inflammation were rarely found. Beyond that, our patient showed extensive preseptal and septal inflammation with proptosis, positive relative afferent pupillary defect, and limited extraocular movement in all directions. However, in term of the management, getting rid of intraocular infection would control infection in both conditions. Because of the high susceptibility to infection in the 
immunocompromised status (active autoimmune disease accompanied with the administration of corticosteroids) and inadequate penetration efficiency of the antibiotic agents into the eye, the infection progressed rapidly even in the hospitalized patient. Although cloxacillin was administered since the patient had skin lesions that were prone to infection, the dosage of the drug was not adequate to pass the blood-brain barrier [29]. Since this organism produces beta-lactamase, it is resistant to first-generation penicillins and cephalosporins. Most isolates are susceptible to fluoroquinolones, third-generation cephalosporins and aminoglycosides [30], thus the main antibiotic treatments for A. hydrophila are fluoroquinolones and cefotaxime [9].

Probably due to the partial antibiotic treatment, the result of two hemocultures was negative, which could not indicate the septicemic status. Laboratory investigations also failed to identify the primary source of infection. However, the patient may develop Aeromonasinduced septicemia from skin lesions which makes the infection spread via the bloodstream into the eye [31]. Even though the patient developed Aeromonas septicemia in the hospital, it was not certain if the acquired organism came from the hospital [17]. On the other hand, exogenous endophthalmitis resulting from minute trauma or adjacent skin infection could be a differential diagnosis even without evidence of traumatic corneal injury or infiltration. Other exogenous sources that have been purposed include hot water from storage tank [32], fish hook injury to the eye during fresh-water fishing [24], and long-dwelling catheter in the immunocompromised patient [27].

A. hydrophila has a variety of virulence factors that contribute to the pathogenesis, such as cytotoxin, protease, and hemolysins. High temperature, as in the human body, will increase cytotoxin and hemolysin activity [33] as well as hypoxic condition which will affect the expression of virulence factors [34]. These parameters might be the factors for rapid progression in our patient who experienced septicemic condition. Until now, there was no report of specific genetic or molecular interactions between Aeromonas and PV.

The diagnosis for endophthalmitis is based on clinical findings. A high index of suspicion is vital for early detection in immunocompromised or high-risk individuals. Once the patient reports clinical signs of blurred vision or any ocular symptoms, the ophthalmic examination should be performed thoroughly for early detection. If the examination is unremarkable, serial examinations must be performed. Once endophthalmitis was diagnosed, species-specific PCR could be used for early detection of the organism [10].

However, this report might have some limitations. As we did not follow the patient from the beginning of infection development, we could not identify if the skin lesions initially showed signs of infection. If there was an active skin pustule or cellulitis, the diagnosis of endogenous endophthalmitis could have been made with the positive culture of the same organism. Polymerase chain reaction could detect organism in negative hemoculture individual [35]. Unfortunately, the analysis was not performed due to financial limitation. We also do not know for sure whether the orbital cellulitis occurred as a consequence or concomitant of endophthalmitis, since clinical presentation revealed both conditions to be severe. Mostly, patients develop endophthalmitis first then progress to panophthalmitis, so we believe that our case followed the same order. However, concomitant infection of endophthalmitis and orbital cellulitis may also occur in septicemic patients. 
Conclusion

Ocular infection in PV is rare, and only fungal and herpetic infection have been reported. A. hydrophila, as an opportunistic infection, can cause fulminant rapidly progressive panophthalmitis in active PV patients who had risk factors contributing to immunocompromised status, which results in a poor visual prognosis. Unfortunately, recurrence of the systemic disease may lead to the same clinical manifestation in the remaining eye. We should be highly suspicious in these patients, since prompt management is required to save the vision.

\section{Acknowledgement}

The authors thank the patient for his consent and participation, the hospital staffs, and Mr. Dylan Southard and Ms. Thippayawan Thanapaisal for assistance with the English language presentation of the manuscript.

\section{Statement of Ethics}

The authors have no ethical conflicts to disclose. Informed consent was obtained from our patients. The study was conducted in accordance with the tenets of the Declaration of Helsinki and was approved by the Khon Kaen University Ethics Committee for Human Research.

\section{Disclosure Statement}

The authors have no financial disclosures. The authors have no conflicts of interest to disclose.

\section{Funding Sources}

The authors have no funding sources to disclose.

\section{Author Contributions}

S.T. and P.T. interpreted the data. S.T. drafted the paper. S.T. and P.T. gave final approval of the published version.

\section{References}

1 Chirinos-Saldaña P, Navas A, Ramírez-Miranda A, Jiménez-Martínez MC, Graue-Hernández EO. Pemphigus: an ophthalmological review. Eye Contact Lens. 2016 Mar;42(2):91-8.

2 Yang HK, Han YK, Wee WR, Lee JH, Kwon JW. Bilateral herpetic keratitis presenting with unilateral neurotrophic keratitis in pemphigus foliaceus: a case report. J Med Case Reports. 2011 Jul;5(1):328.

3 Takeshita T. Bilateral herpes simplex virus keratitis in a patient with pemphigus vulgaris. Clin Exp Dermatol. 1996 Jul;21(4):291-2. 
Thanapaisal and Thariya: Aeromonas hydrophila Panophthalmitis in Pemphigus Vulgaris: A Case Report

4 Smith RJ, Manche EE, Mondino BJ. Ocular cicatricial pemphigoid and ocular manifestations of pemphigus vulgaris. Int Ophthalmol Clin. 1997;37(2):63-75.

5 Akhyani M, Keshtkar-Jafari A, Chams-Davatchi C, Lajevardi V, Beigi S, Aghazadeh N, et al. Ocular involvement in pemphigus vulgaris. J Dermatol. 2014 Jul;41(7):618-21.

6 Daoud YJ, Cervantes R, Foster CS, Ahmed AR. Ocular pemphigus. J Am Acad Dermatol. 2005 Oct;53(4):58590.

7 España A, Iranzo P, Herrero-González J, Mascaro JM Jr, Suárez R. Ocular involvement in pemphigus vulgaris a retrospective study of a large Spanish cohort. J Dtsch Dermatol Ges. 2017 Apr;15(4):396-403.

8 Tan JC, Tat LT, Francis KB, Mendoza CG, Murrell DF, Coroneo MT. Prospective study of ocular manifestations of pemphigus and bullous pemphigoid identifies a high prevalence of dry eye syndrome. Cornea. 2015 Apr;34(4):443-8.

9 Parker JL, Shaw JG. Aeromonas spp. clinical microbiology and disease. J Infect. 2011 Feb;62(2):109-18.

10 Igbinosa IH, Igumbor EU, Aghdasi F, Tom M, Okoh AI. Emerging Aeromonas species infections and their significance in public health. ScientificWorldJournal. 2012;2012:625023.

11 King GE, Werner SB, Kizer KW. Epidemiology of Aeromonas infections in California. Clin Infect Dis. 1992 Sep;15(3):449-52.

12 Sen K, Rodgers M. Distribution of six virulence factors in Aeromonas species isolated from US drinking water utilities: a PCR identification. J Appl Microbiol. 2004;97(5):1077-86.

13 Havelaar AH, Versteegh JF, During M. The presence of Aeromonas in drinking water supplies in The Netherlands. Zentralbl Hyg Umweltmed. 1990 Sep;190(3):236-56.

14 Dixon B, Presley SM, Rainwater TR, Austin GP, Platt SG, Zak JC, et al. Natural disaster microbiology. Microbe. 2008;3:312-3.

15 Presley SM, Rainwater TR, Austin GP, Platt SG, Zak JC, Cobb GP, et al. Assessment of pathogens and toxicants in New Orleans, LA following Hurricane Katrina. Environ Sci Technol. 2006 Jan;40(2):468-74.

16 Ramalivhana JN, Obi CL, Samie A, Labuschagne C, Weldhagen GF. Random amplified polymorphic DNA typing of clinical and environmental Aeromonas hydrophila strains from Limpopo province, South Africa. Health Popul Nutr. 2010 Feb;28(1):1-6.

17 Ko WC, Lee HC, Chuang YC, Liu CC, Wu JJ. Clinical features and therapeutic implications of 104 episodes of monomicrobial Aeromonas bacteraemia. J Infect. 2000 May;40(3):267-73.

18 Altwegg M, Geiss HK, Freij BJ. Aeromonas as a human pathogen. Crit Rev Microbiol. 1989;16(4):253-86.

19 Clark NM, Chenoweth CE. Aeromonas infection of the hepatobiliary system: report of 15 cases and review of the literature. Clin Infect Dis. 2003 Aug;37(4):506-13.

20 Gonçalves JR, Brum G, Fernandes A, Biscaia I, Correia MJ, Bastardo J. Aeromonas hydrophila fulminant pneumonia in a fit young man. Thorax. 1992 Jun;47(6):482-3.

21 Kao HT, Huang YC, Lin TY. Fatal bacteremic pneumonia caused by Aeromonas hydrophila in a previously healthy child. J Microbiol Immunol Infect. 2003 Sep;36(3):209-11.

22 Sire JM, Ropert P, Donnio PY. Aeromonas hydrophila blepharoconjunctivitis. Eur J Clin Microbiol Infect Dis. 1990 Dec;9(12):904-5.

23 Smith JA. Ocular Aeromonas hydrophila. Am J Ophthalmol. 1980 Mar;89(3):449-51.

24 Cohen KL, Holyk PR, McCarthy LR, Peiffer RL. Aeromonas hydrophila and Plesiomonas shigelloides endophthalmitis. Am J Ophthalmol. 1983 Sep;96(3):403-4.

25 Tamura T, Hida T. [A case of endogenous Aeromonas hydrophila endophthalmitis]. Nippon Ganka Gakkai Zasshi. 2003 Sep;107(9):535-7. Japanese.

26 Khan MI, Walters G, Metcalfe T. Bilateral endogenous endophthalmitis caused by Aeromonas hydrophila. Eye (Lond). 2007 Sep;21(9):1244-5.

27 Sohn HJ, Nam DH, Kim YS, Paik HJ. Endogenous aeromonas hydrophila endophthalmitis in an immunocompromised patient. Korean J Ophthalmol. 2007 Mar;21(1):45-7.

28 Chou SY, Tsai CC, Kau SC, Kau HC, Hsu WM. Aeromonas hydrophila orbital cellulitis in a patient with myelodysplastic syndrome. J Chin Med Assoc. 2004 Jan;67(1):51-3.

29 World Health Organization. WHO model prescribing information: drugs used in bacterial infections [Internet]. 2000 [cited 2019 Feb 5]. Available from: http://apps.who.int/medicinedocs/pdf/s5406e/s5406e.pdf

30 Jameson J, Fauci AS, Kasper DL, Hauser SL, Longo DL, Loscalzo J. Harrison's principles of internal medicine [Internet]. 20th ed. New York: McGraw-Hill; 2018. [cited 2019 Feb 21]. Available from: https://accessmedicine. $m$ hmedical.com/content.aspx?bookid=2129\&sectionid=159213747

31 Mukhopadhyay C, Chawla K, Sharma Y, Bairy I. Emerging extra-intestinal infections with Aeromonas hydrophila in coastal region of southern Karnataka. J Postgrad Med. 2008 Jul-Sep;54(3):199-202.

32 Ryan L, Higgins G, Doyle M. Aeromonas species endogenous endophthalmitis. JMM Case Rep. 2017 May;4(5):e005094.

33 Yu HB, Zhang YL, Lau YL, Yao F, Vilches S, Merino S, et al. Identification and characterization of putative virulence genes and gene clusters in Aeromonas hydrophila PPD134/91. Appl Environ Microbiol. 2005 Aug;71(8):4469-77. 


\section{Case Reports in Ophthalmology}

34 Rasmussen-Ivey CR, Figueras MJ, McGarey D, Liles MR. Virulence factors of Aeromonas hydrophila: in the wake of reclassification. Front Microbiol. 2016 Aug;7(7):1337.

35 Warhurst G, Maddi S, Dunn G, Ghrew M, Chadwick P, Alexander P, et al. Diagnostic accuracy of SeptiFast multi-pathogen real-time PCR in the setting of suspected healthcare-associated bloodstream infection. Intensive Care Med. 2015 Jan;41(1):86-93.

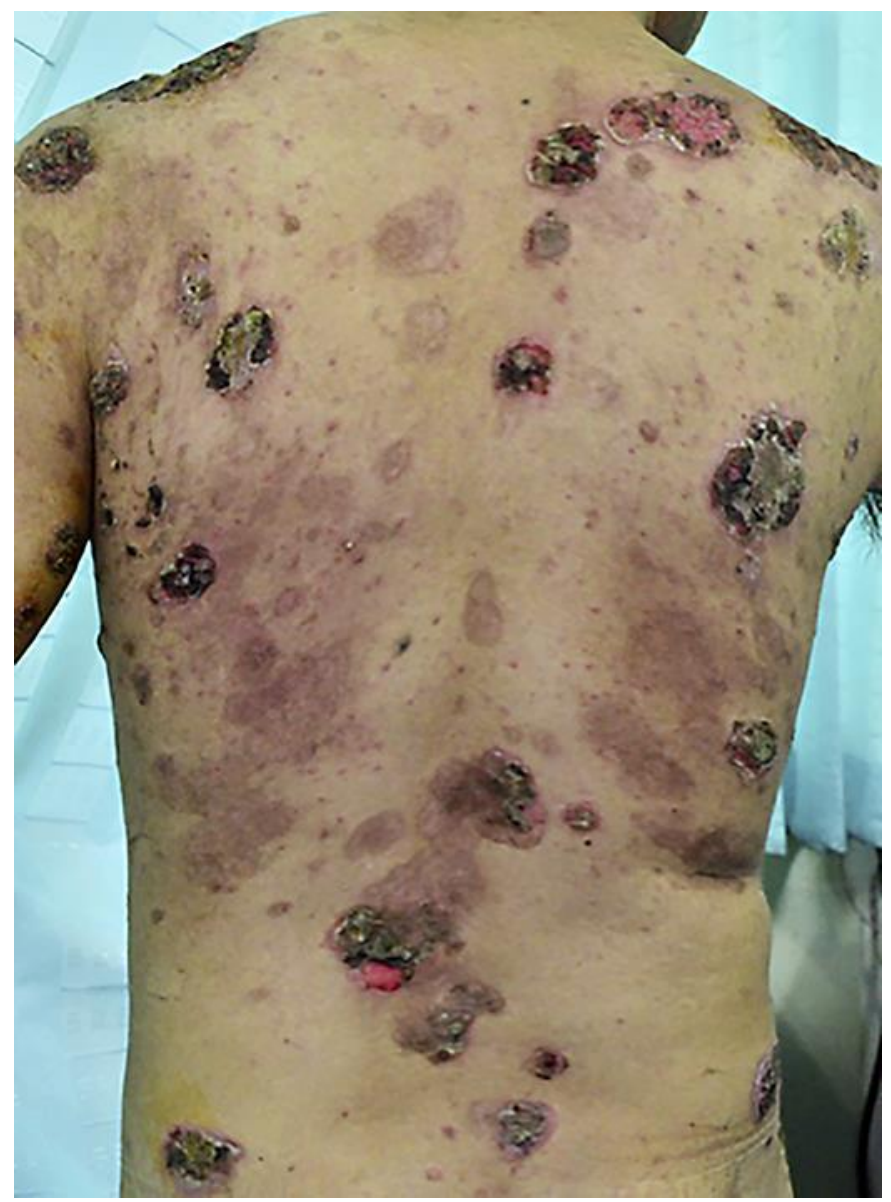

Fig. 1. Skin lesions exhibiting multiple discrete flaccid blisters and oval-shaped erosions covered with hemorrhagic crust, distributed along the trunk and extremities. Some of the lesions were healed as postinflammatory hyperpigmented patches. 


\section{Case Reports in Ophthalmology}

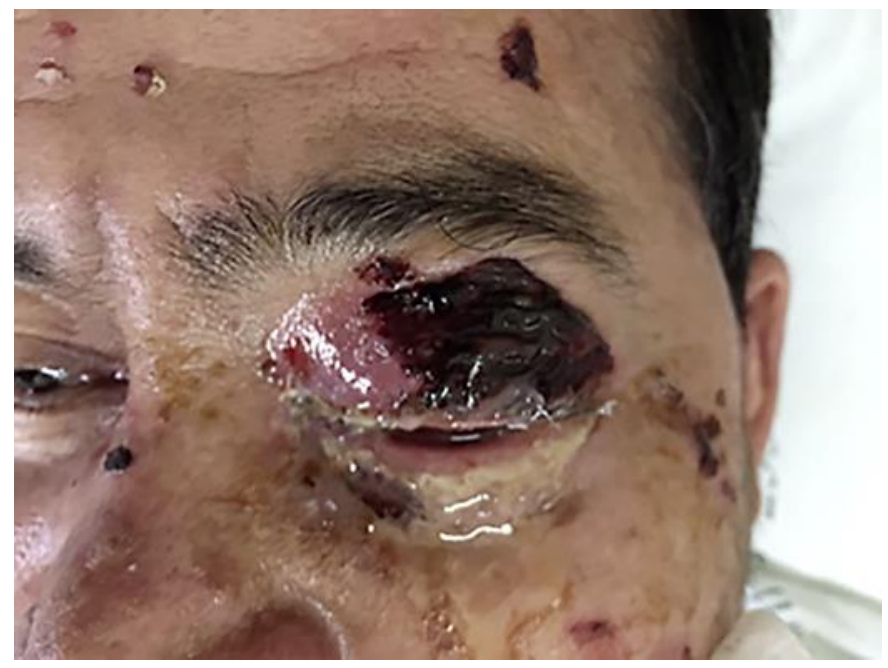

Fig. 2. Periorbital soft tissue inflammation with hyperpigmented flaccid bullous at the left upper eyelid.

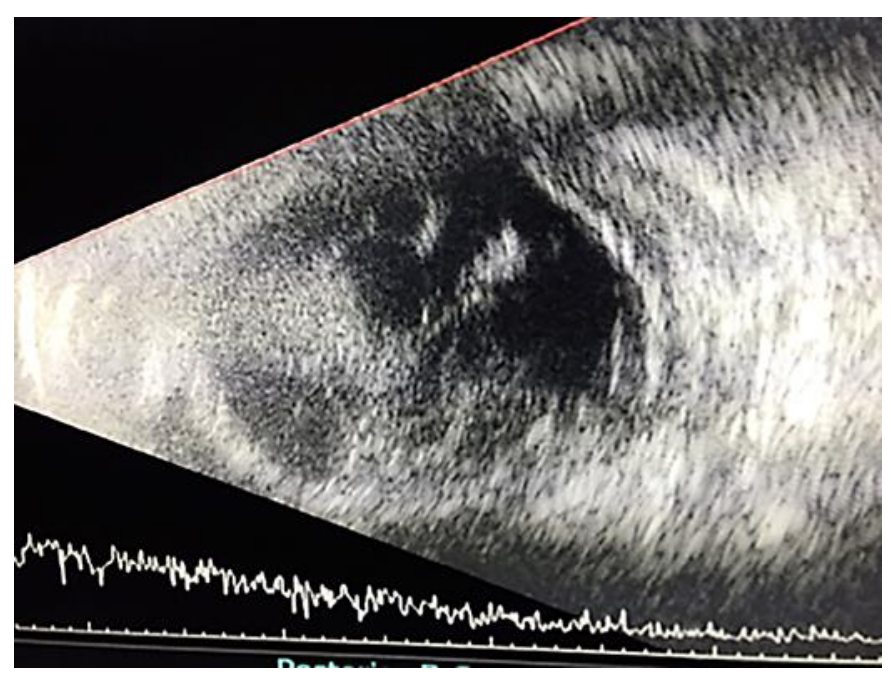

Fig. 3. Ultrasonography showing marked generalized opacity of the vitreous and chorioretinal thickening with no evidence of retinal abscess. 


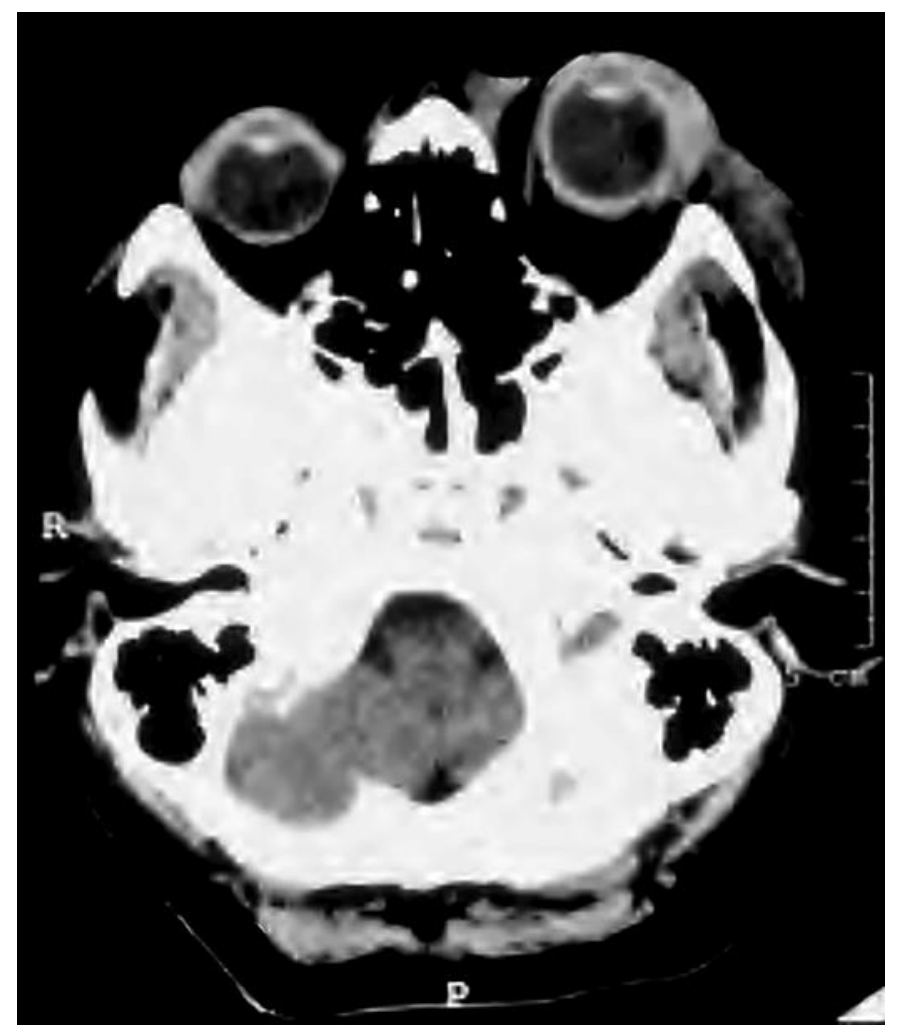

Fig. 4. Computed tomography scan demonstrating left proptosis with periorbital tissue swelling, diffused scleral thickening, and retrobulbar fat stranding. The paranasal sinuses were normal and no subperiosteal abscess was documented. 